Supporting Information for

\title{
Revealing mitochondrial microenvironmental evolution triggered by photodynamic therapy
}

Jing Yue ${ }^{\dagger}$, Yanting Shen ${ }^{\dagger}$, Lijia Liang ${ }^{\dagger}$, Lili Cong ${ }^{\dagger}$, Weiqing $\mathrm{Xu}^{\dagger}$, Wei Shi ${ }^{\S}$, Chongyang Liang ${ }^{\ddagger}$, Shuping $\mathrm{Xu}^{* \dagger}$

'State Key Laboratory of Supramolecular Structure and Materials, Institute of Theoretical Chemistry, College of Chemistry, Jilin University, Changchun, 130012, China.

Institute of Frontier Medical Science, Jilin University, Changchun, 130021, China.

${ }^{\S}$ Key Lab for Molecular Enzymology and Engineering of Ministry of Education, Jilin University, Changchun, 130012, China.

* Corresponding author

Email:xusp@jlu.edu.cn (X.S.) 
Table of contents

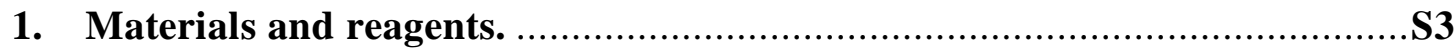

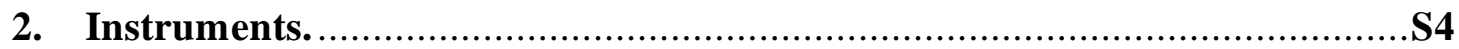

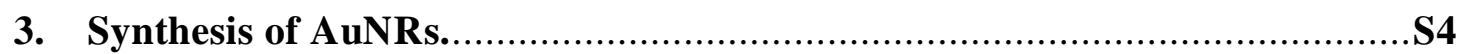

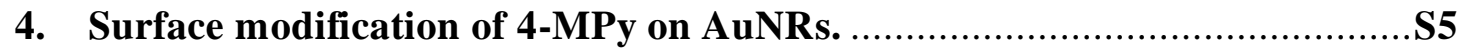

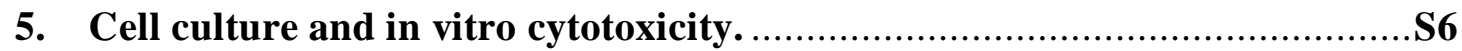

6. Figure S1: Measurements of the DCFH fluorescence density........................

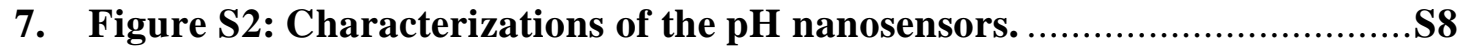

8. Figure S3: pH Calibration of the pH nanosensors. ...................................

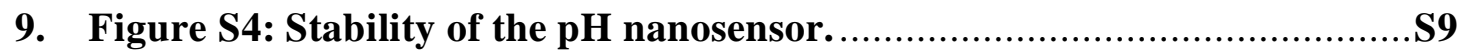

10. Figure S5: I1003/I1095 of pH nanosensors in mitochondria. ...................S10

11. Table S1: The peak assignments of the SERS spectrum of 4-MPy...........S10

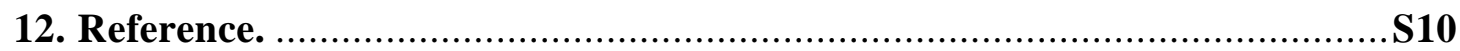




\section{Materials and reagents}

Tetrachloroauric acid trihydrate $\left(\mathrm{HAuCl} 4 \cdot 3 \mathrm{H}_{2} \mathrm{O}\right)$, cetyltrimethylammonium bromide (CTAB) and 4-mercaptopyridine (4MPy) were purchased from Aladdin Industrial Corporation. Ascorbic acid was obtained from Beijing Chemical Company. $\mathrm{AgNO}_{3}$ was purchased from Shanghai Chemical Company. $\mathrm{NaBH}_{4}$ was obtained from Tianjin Fuchen Chemical Reagents Factory. Chlorin e6 (Ce6) and Mito-tracker Red was purchased from Sigma. Mitochondria location peptides (MLS, MLALLGWWWFFSRKKC) were bought from Apeptide (Shanghai). Dulbecco' modified Eagle's medium WST-1 was obtained from Hoffmann-La Roche LTD. Culture medium Dulbecco's Modification of Eagle's Medium (DMEM) and fetal bovine serum (FBS) were purchased from Thermo Fisher Scientific. $\mathrm{Na}_{2} \mathrm{HPO}_{4}, \mathrm{NaH}_{2} \mathrm{PO}_{4}, \mathrm{NaOH}$ and $\mathrm{HCl}$ were obtained from Beijing Chemical Factory. Mitochondrial Membrane Potential Assay Kit with JC-1 and DCFH-DA fluorescence dyes were purchased from BestBio biotechnologies Co. Ltd. (Shanghai, China). MCF-7 (human breast cancer cell line), HepG2 (human liver hepatocellular carcinoma cell line) and L02 (human normal hepatocytes cell line) cells were bought from Shanghai ATCC cell bank who have been issued the permission of the Human Research Ethics Committee of the country for manipulations of human's cells. 


\section{Instruments}

JEM-2100F field emission transmission electron microscope (TEM, JEOL, Tokyo, Japan) was used for characterizing the morphology of the prepared gold nanorods (AuNRs). Ultraviolet-visible (UV-vis) spectra were collected using an Ocean Optics USB4000 spectrometer. SERS spectra were obtained using a confocal Raman system (LabRAM Aramis, Horiba JobinYvon, USA) with a He-Ne $(632.8 \mathrm{~nm})$ laser as the excitation source. Confocal fluorescent images were collected using a FV1000 confocal fluorescence microscope (Olympus). The self-built $650 \mathrm{~nm}$ light-emitting diode (LED) lamp (a Petri dish was placed inside a box with the LED, the distance between LED and the Petri dish is about $25 \mathrm{~cm}$. The power at the position of Petri dish is about $18 \mathrm{~mW} / \mathrm{cm}^{2}$ ) was employed to produce a ROS.

\section{Synthesis of AuNRs}

Here, AuNRs which were stabilized by cetyltrimethylammonium bromide (CTAB) were synthesized by a seed-mediated growth method ${ }^{1,2}$ First of all, $5.0 \mathrm{~mL}$ of $0.5 \mathrm{mM} \mathrm{HAuCl}_{4}$ was added to the $5.0 \mathrm{~mL}$ of a $0.2 \mathrm{M} \mathrm{CTAB}$ solution, and then $600 \mu \mathrm{L} 0.01 \mathrm{M}$ of ice-cold $\mathrm{NaBH}_{4}$ was added to the mixture under stirring for $2 \mathrm{~min}$. The mixture was aging for $2 \mathrm{~h}$ at the $30{ }^{\circ} \mathrm{C}$ to obtain the Au seeds. Then $60 \mu \mathrm{L}$ of the seed solution was added to a mixture containing $5.0 \mathrm{~mL}$ of $0.2 \mathrm{M} \mathrm{CTAB}$ aqueous solution, $25 \mathrm{~mL}$ of 1.0 $\mathrm{mM} \mathrm{HAuCl}_{4}, 650 \mu \mathrm{L}$ of $4.0 \mathrm{mM} \mathrm{AgNO}_{3}$, and $350 \mu \mathrm{L}$ of $78.8 \mathrm{mM}$ ascorbic 
acid. The mixture was kept undisturbed for $12 \mathrm{~h}$ at $30^{\circ} \mathrm{C}$.

\section{Surface modification of 4-MPy on AuNRs.}

Before modification of AuNRs, two times centrifugation at $6000 \mathrm{rpm}$ for 10 min were followed to remove the excessive $\mathrm{CTAB}$ and the precipitates were redispersed in water. Transmission electron microscope (TEM), ultraviolet-visible (UV-Vis, Ocean Optics, USB4000) and dynamic light scattering (DLS, Malvern Zetasizer Nano ZS) spectroscopies were used to measure the size, morphology, plasmonic property and zeta potential of the obtained AuNRs.

Then the AuNRs were modified with 4-MPy to enable the $\mathrm{pH}$ sensing. Briefly, $1 \mu \mathrm{L}$ of a $1.0 \mathrm{mM}$ 4-MPy methanol solution was added to $1.0 \mathrm{~mL}$ of $1.0 \mathrm{nM}$ AuNRs solution and the mixture was incubated at room temperature for $4 \mathrm{~h}$. Then, extra 4-MPy were removed by centrifugation (4000 rpm, $8 \mathrm{~min}$ ) and the AuNRs-MPy sensors were obtained. To further achieve specific mitochondria targeting functions, the nanoprobes were subsequently modified with MLS (with and without FITC) to obtain $\mathrm{pH}$ nanosensoers. The mixtures were incubated at room temperature overnight and the linking between the peptides and AuNRs are based on a traditional surface reaction of the mercapto-group of peptides by forming S-Au bond. UV-Vis spectroscopy and DLS were used to characterize the nanoprobes. 


\section{Cell culture and in vitro cytotoxicity.}

Cells were cultured in a culture dish in Dulbecco's Modified Eagle's Medium (DMEM, Thermo Fisher Scientific) plus 10\% fetal bovine serum (FBS, Thermo Fisher Scientific) at $37{ }^{\circ} \mathrm{C}$ in a humidified environment with $5 \%$ of $\mathrm{CO}_{2}$ for $24 \mathrm{~h}$. The in vitro cytotoxicity of AuNRs-MLS-Mpy $\mathrm{pH}$ nanosensors was assessed by the WST-1 (2-(4-Iodophenyl)-3-(4nitrophenyl)-5-(2,4-disulfophenyl)-2H-tetrazolium, monosodium salt) assay. Cells were firstly seeded with a density of $10^{4}$ cells per well to 96well plates with $100 \mu \mathrm{L}$ of the DMEM containing $10 \%$ FBS at $37^{\circ} \mathrm{C}$ in a $5 \% \mathrm{CO}_{2}$ incubator for $24 \mathrm{~h}$. After the successful cell attachment by one day incubation, cells were incubated with fresh culture medium containing 0.1 $\mathrm{nM}$ of nanoprobes for another $12 \mathrm{~h}$. Afterwards, the cells were rinsed with PBS buffer, and then we added $20 \mu \mathrm{L}$ of the WST- 1 solution into each well and incubated them for another $2 \mathrm{~h}$. At last, the absorption intensity of each well was measured at $450 \mathrm{~nm}$ by a microplate reader (Tecan Sunrise). Cells incubated with the standard cell culture medium were used for cell viability evaluation as the control group.

\section{Measurements of the DCFH fluorescence density.}

Image $\mathbf{J}$ software was used to calculate the average density of DCFH fluorescence dyes in three kinds of cell lines after they were treated with $1.2 \mu \mathrm{M}$ of Ce6 and $0.1 \mathrm{nM} \mathrm{pH}$ nanosensors exposed to a $650 \mathrm{~nm}$ LED (18 $\mathrm{mW} / \mathrm{cm}^{2}$ ) for $0,1,3,5$ and $7 \mathrm{~min}$, respectively. First, the optical image was 
converted to an 8-bit image. Next, four options in Area, Min \& max gray value, Integrated density and \%Area were selected in set measurement of analysis, respectively. Next, the threshold of the unit in image was adjust as 0.01 , and then the final intensity statistics results were obtained by Measure in the Analyze function. Subsequently, the Average Fluorescence Density values of 3 groups were obtained by dividing Integrated density by Area respectively.

(a)

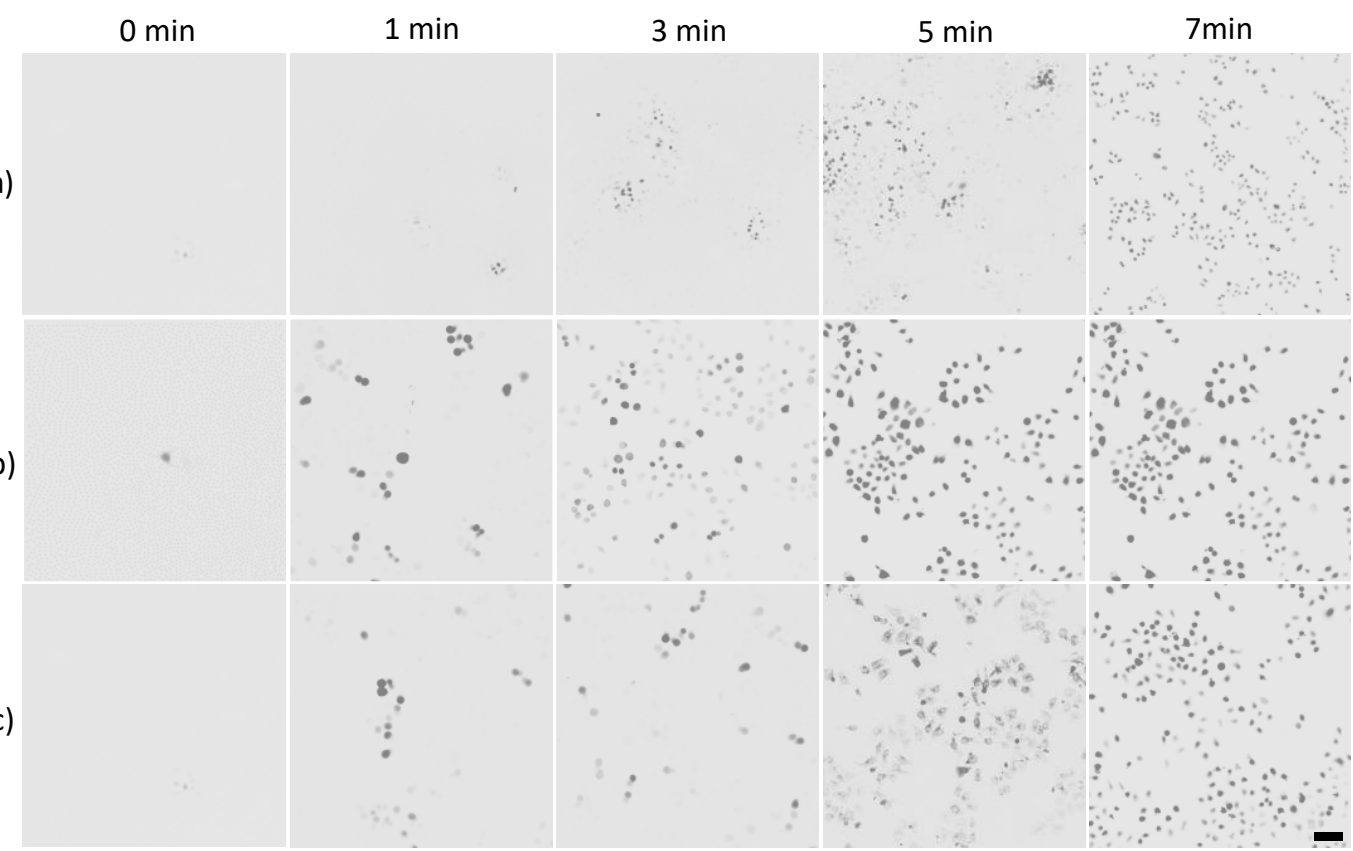

Figure S1. The images of Figure 1 after processing with Image J software. (a) MCF-7, (b) HepG2 and (c) LO2 cells. (e) The average fluorescence density of DCFH-DA in MCF-7, HepG2 and LO2 cells after they were treated for different time. 


\section{Characterizations of the $\mathrm{pH}$ nanosensors}
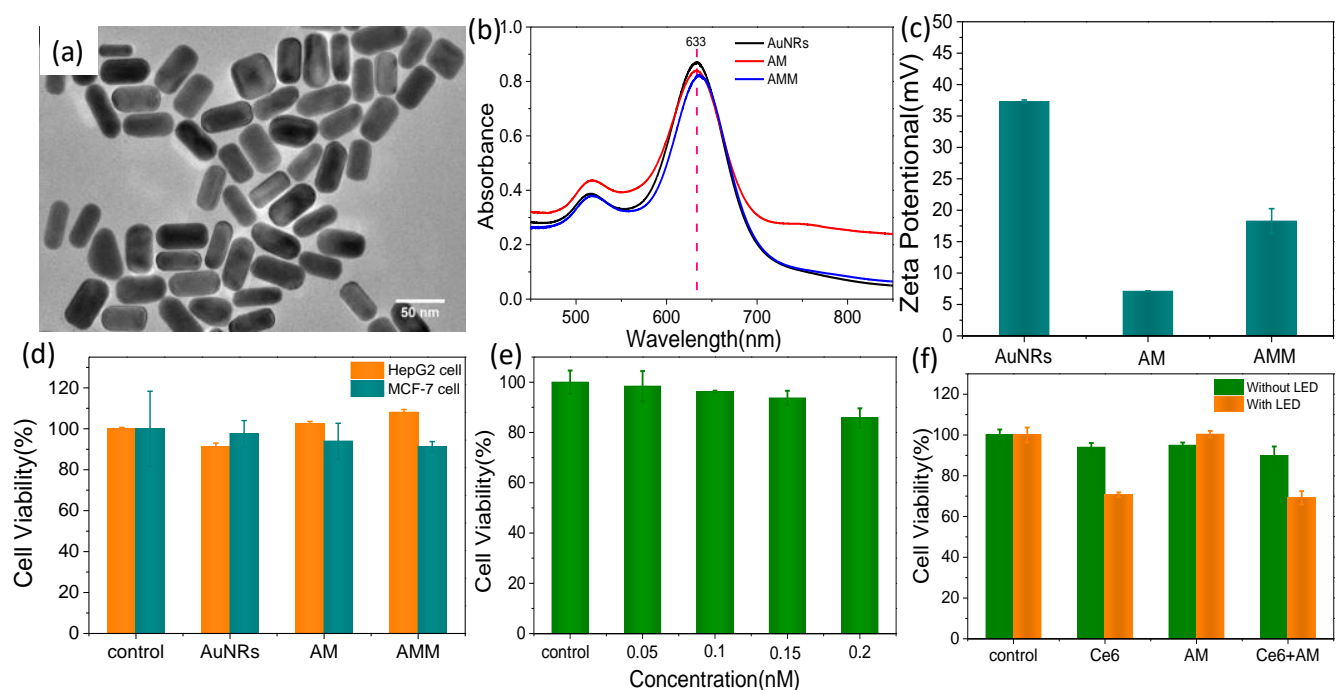

Figure S2. (a) TEM image of AuNRs. (b) UV-vis spectra and (c) Zeta potential of AuNRs, AuNRs-MPy and AuNRs-MPy-MLS pH nanosensor. (d) Cell viabilities of HepG2 and MCF-7 cells incubated with 0.1nM of AuNRs, AuNRs-MPy and AuNRsMPy-MLS for $24 \mathrm{~h}$. (e) Cell viabilities of HepG2 cells incubated with different concentrations of $\mathrm{pH}$ nanosensor for $24 \mathrm{~h}$. (f) Cell viabilities of HepG2 cells incubated with $1.2 \mu \mathrm{M} \mathrm{Ce} 6,0.1 \mathrm{nM} \mathrm{pH}$ nanosensors, $\mathrm{Ce} 6$ and $\mathrm{pH}$ nanosensors for $12 \mathrm{~h}$ before and after the irradiation of a $650 \mathrm{~nm}$ lamp $\left(18 \mathrm{~mW} / \mathrm{cm}^{2}\right)$ for $3 \mathrm{~min}$. 


\section{8. pH Calibration}

(a)

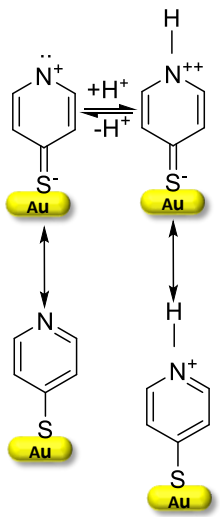

(b)

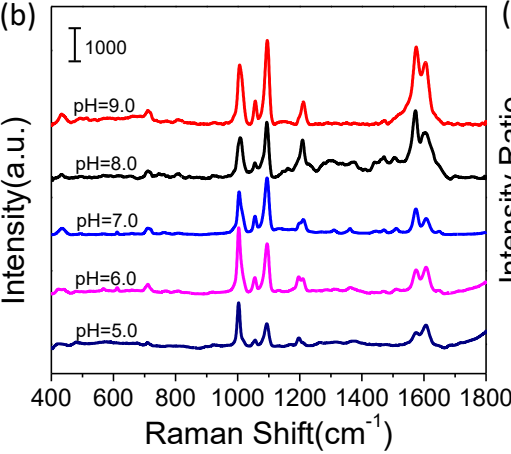

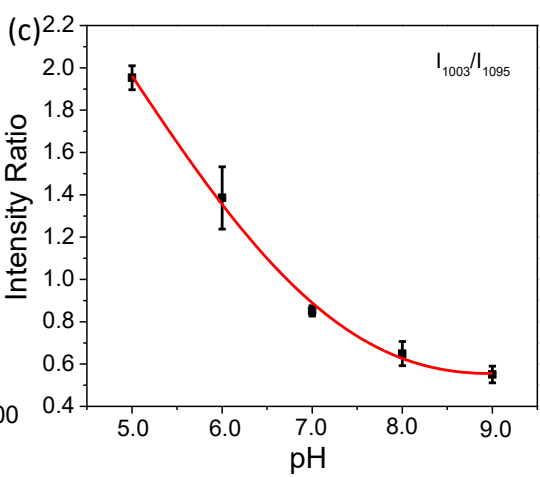

Figure S3 (a) Resonance structures and ionization behavior of 4-MPy on AuNRs. (b) SERS spectra of nanosensors in culture medium with various $\mathrm{pH}$ values in the spectral range of 400$1800 \mathrm{~cm}^{-1} . \lambda \mathrm{ex}=632.8 \mathrm{~nm}, \mathrm{t}=10 \mathrm{~s}$ and accumulations $=2$ times. (c) The intensity ratio of 1003 $\mathrm{cm}^{-1} / 1095 \mathrm{~cm}^{-1}$ as a function of $\mathrm{pH}$ values.

\section{Stability of the pH nanosensor}
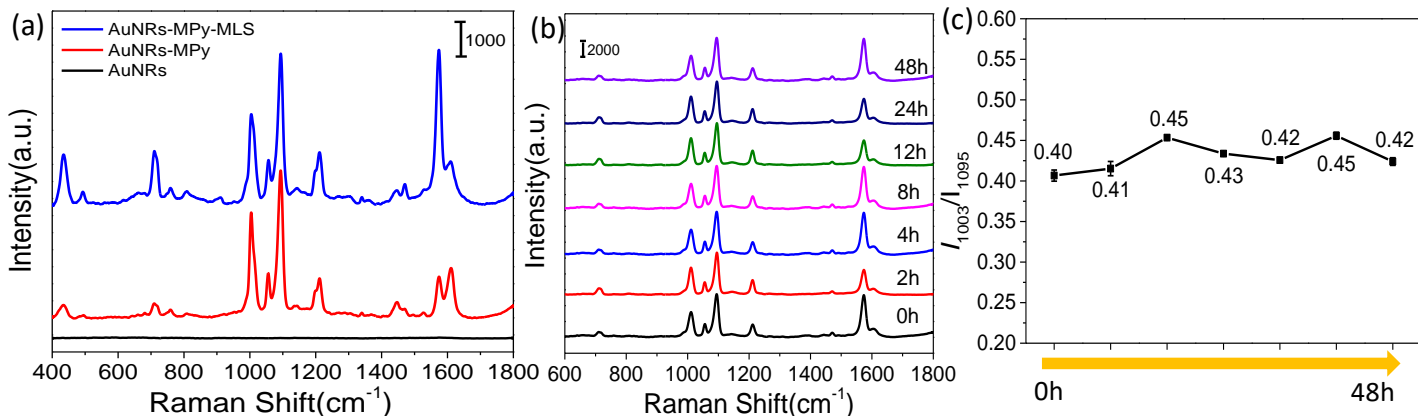

Figure S4. SERS spectra of (a) AuNRs, AuNRs-MPy and AuNRs-MPy-MLS pH probes and (b) $\mathrm{pH}$ nanosensor in culture medium for different time $(0,2,4,8,12,24$ and 48 h). (c) The I1003/I 1095 of $\mathrm{pH}$ nanosensors after incubated with cell culture medium for different time. 
10. $\mathrm{I}_{1003} / \mathrm{I}_{1095}$ of $\mathrm{pH}$ nanosensors in mitochondria.
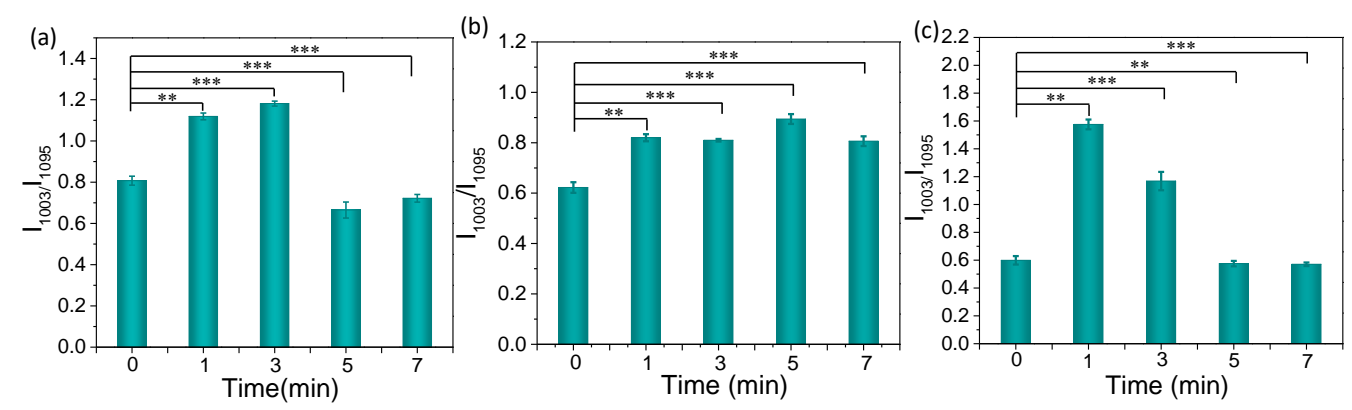

Figure S5. I1003/I1095 of pH nanosensors in mitochondria of (a) MCF-7 (b) HepG2 and (c) LO2 cells at different irradiation time.

\section{The peak assignments of the SERS spectrum of 4-MPy.}

Table S1. The peak assignments of the SERS spectrum of 4-MPy ${ }^{[2]}$.

\begin{tabular}{cc}
\hline Raman shift $\left(\mathbf{c m}^{-1}\right)$ & Assignment \\
\hline 428 & $\mathrm{~s}(\mathrm{C}-\mathrm{S}) / \mathrm{g}(\mathrm{CCC})$ \\
708 & $\mathrm{~b}(\mathrm{CC}) / \mathrm{n}(\mathrm{C}-\mathrm{S})$ \\
1003 & Ring breathing \\
1095 & Ring breathing/C-S \\
1199 & $\mathrm{C}-\mathrm{H}$ in-plane bending/wagging \\
1576 & $\mathrm{n}(\mathrm{C} / \mathrm{C} / \mathrm{C} / \mathrm{N})$ \\
1608 & $\mathrm{n}(\mathrm{C}=\mathrm{C})$ \\
\hline
\end{tabular}

\section{Reference.}

(1) Bi, X.; Du, X.; Jiang, J.; Huang, X. Facile and Sensitive Glucose Sandwich Assay Using In Situ-Generated Raman Reporters. Anal.Chem., 2015, 87, 2016-2021.

(2) Zheng, X.-S.; Hu, P.; Cui, Y.; Zong, C.; Feng, J.-M.; Wang, X.; Ren, B. BSA-coated nanoparticles for improved SERS-based intracellular pH sensing. Anal. Chem., 2014, $86,12250-12257$. 